\title{
Axitinib treatment in advanced RAl-resistant differentiated thyroid cancer (DTC) and refractory medullary thyroid cancer (MTC)
}

\author{
Jaume Capdevila1, José Manuel Trigo², Javier Aller ${ }^{3}$, José Luís Manzano4, Silvia García Adrián ${ }^{5}$, \\ Carles Zafón Llopis ${ }^{6}$, Òscar Reig ${ }^{7}$, Uriel Bohn ${ }^{8}$, Teresa Ramón y Cajal ${ }^{9}$, Manuel Duran-Poveda $^{10}$, \\ Beatriz González Astorga ${ }^{11}$, Ana López-Alfonso ${ }^{12}$, Javier Medina Martínez ${ }^{13}$, Ignacio Porras ${ }^{14}$, \\ Juan Jose Reina ${ }^{15}$, Nuria Palacios ${ }^{16}$, Enrique Grande ${ }^{17}$, Elena Cillán ${ }^{18}$, Ignacio Matos ${ }^{19}$ and Juan Jose Grau ${ }^{20}$
}

${ }^{1}$ Medical Oncology Department, Gastrointestinal and Endocrine Tumor Unit, Vall d'Hebron University Hospital, Universitat Autònoma de Barcelona, Barcelona, Spain, ${ }^{2}$ Medical Oncology Department, University Hospital Virgen de la Victoria, Málaga, Spain,

${ }^{3}$ Endocrinology Department, University Hospital Puerta de Hierro, Madrid, Spain, ${ }^{4}$ Medical Oncology Department, Catalan Oncology Institute (ICO-Badalona), University Hospital Germans Trias y Pujol, Barcelona, Spain, ${ }^{5}$ Medical Oncology Department, University Hospital of Móstoles, Móstoles, Madrid, Spain, ${ }^{6}$ Endocrinology and Nutrition Department, Vall d'Hebron University Hospital, Barcelona, Spain, ${ }^{7}$ Medical Oncology Department, Translational Genomics and Targeted Therapeutics in Solid Tumors (IDIBAPS), Hospital Clínic of Barcelona, Barcelona, Spain, ${ }^{8}$ Medical Oncology Department, University Hospital of Gran Canaria Doctor Negrín, Las Palmas, Spain, ${ }^{9}$ Medical Oncology Department, University Hospital of Santa Creu i Sant Pau, Barcelona, Spain, ${ }^{10}$ General and Endocrine Surgery Department, University Hospital Rey Juan Carlos, Madrid, Spain, ${ }^{11}$ Medical Oncology Department, University Hospital Virgen de las Nieves, Granada, Spain, ${ }^{12}$ Medical Oncology Department, University Hospital Infanta Leonor, Madrid, Spain, ${ }^{13}$ Medical Oncology Department, Hospital Virgen de la Salud, Toledo, Spain, ${ }^{14}$ Medical Oncology Department, University Hospital Reina Sofía, Córdoba, Spain, ${ }^{15}$ Medical Oncology Department, University Hospital Virgen Macarena, Sevilla, Spain, ${ }^{16}$ Endocrinology Department, University Hospital Puerta de Hierro, Madrid, Spain, ${ }^{17}$ Medical Oncology Department, University Hospital Ramón y Cajal, Madrid, Spain, ${ }^{18}$ Medical Oncology Department, University Hospital of Santa Creu i Sant Pau, Barcelona, Spain, ${ }^{19}$ Medical Oncology Department, Gastrointestinal and Endocrine Tumor Unit, Val d'Hebron University Hospital, Universitat Autònoma de Barcelona, Barcelona, Spain, and ${ }^{20}$ Medical Oncology Department, University of Barcelona, Hospital Clínic of Barcelona, Barcelona, Spain

Correspondence should be addressed to J Capdevila Email jacapdevila@vhebron.net

\section{Abstract}

Background: Axitinib, an antiangiogenic multikinase inhibitor (MKI), was evaluated in the compassionate use programme (CUP) in Spain (October 2012-November 2014).

Subjects and Methods: 47 patients with advanced radioactive iodine (RAI)-refractory differentiated thyroid cancer (DTC, $n=34$ ) or medullary thyroid cancer (MTC, $n=13$ ) with documented disease progression were treated with axitinib $5 \mathrm{mg}$ b.i.d. The primary efficacy endpoint was objective response rate (ORR) by Response Evaluation Criteria In Solid Tumors (RECIST) v1.1. Progression-free survival (PFS) and adverse events (AEs) were secondary objectives. Regulatory authorities validated the CUP, and all patients signed informed consent form.

Results: Axitinib was administered as first-line therapy in 17 patients (36.2\%), as second-line in 18 patients (38.3\%) and as third/fourth-line in 12 patients (25.5\%). With a median follow-up of 11.5 months (0-24.3), ORR was 27.7\% (DTC: 29.4\% and MTC: $23.1 \%$ ) and median PFS was 8.1 months (95\% Cl: 4.1-12.2) (DTC: 7.4 months (95\% Cl: 3.1-11.8) and MTC: 9.4 months (95\% Cl: 4.8-13.9)). Better outcomes were reported with first-line axitinib, with an ORR of $53 \%$ and a median PFS of 13.6 months compared with $16.7 \%$ and 10.6 months as second-line treatment. Twelve (25.5\%) patients required dose reduction to $3 \mathrm{mg}$ b.i.d. All-grade AEs included asthenia (53.2\%), diarrhoea (36.2\%), hypertension (31.9\%) and mucositis (29.8\%); grade 3/4 AEs included anorexia (6.4\%), diarrhoea (4.3\%) and cardiac toxicity (4.3\%).

Conclusion: Axitinib had a tolerable safety profile and clinically meaningful activity in refractory and progressive thyroid cancer regardless of histology as first-line therapy. To our knowledge, this is the first time that cross-resistance between MKIs is suggested in thyroid cancer, highlighting the importance of prospective sequential clinical studies.

European Journal of Endocrinology

(2017) 177, 309-317

(C) 2017 European Society of Endocrinology Printed in Great Britain
Published by Bioscientifica Ltd. 


\section{Implications for practice}

Antiangiogenic multikinase inhibitors (MKIs) are frequently used to treat radioactive iodine (RAI)-refractory differentiated thyroid cancer (DTC) or medullary thyroid cancer (MTC) refractory to standard surgery or locoregional therapies. Axitinib, an antiangiogenic MKI, was tolerable and provided promising clinical efficacy to patients with refractory and progressive thyroid cancer regardless of tumour histology (DTC or MTC). Better outcomes were reported when axitinib was administered as a first-line treatment, suggesting cross-resistance between MKIs in thyroid cancer and highlighting the importance of prospective sequential clinical studies (Table 1).

\section{Learning objectives:}

- Delineate the efficacy and safety profile of the MKI axitinib in patients with refractory thyroid cancer.

- Recognize the potential for improved clinical outcomes when patients with refractory thyroid cancer receive first-line MKI axitinib.

- Understand the possibility of cross-resistance between MKIs in thyroid cancer.

\section{Introduction}

Thyroid cancer was diagnosed in approximately 298,000 individuals worldwide and resulted in about 40000 deaths in 2012 (1). In Europe there were 63000 cases of thyroid cancer with 7500 deaths. The main types of thyroid carcinoma by histology are differentiated thyroid carcinoma (DTC), medullary thyroid carcinoma (MTC) and anaplastic thyroid carcinoma (ATC), with DTC being the most frequent.

Thyroid tumours have elevated levels of vascular endothelial growth factor (VEGF) and increased angiogenic processes (2). Several multikinase inhibitors (MKIs) targeting the VEGF pathway have shown significant efficacy in advanced and refractory thyroid cancer, corroborating that targeting angiogenesis is an appropriate therapeutic strategy in these tumours. Approved therapeutic agents include cabozantinib and vandetanib for progressive or symptomatic unresectable MTC, and sorafenib and lenvatinib for radioactive iodine (RAI)-refractory DTC. Current treatment guidelines recommend antiangiogenic MKIs for the treatment of patients with metastatic DTC refractory to RAI therapy and locally recurrent or unresectable metastatic MTC $(3,4,5)$.

Axitinib is an oral, potent and selective MKI of VEGF receptors (VEGFRs) 1, 2 and 3. This drug is currently approved for the treatment of advanced renal cell carcinoma (RCC) after failure of prior systemic therapy (sunitinib or cytokine) and also has been tested as second-line therapy in hepatocellular carcinoma and non-small cell lung cancer $(6,7,8)$. Two phase II studies evaluating the efficacy and safety of axitinib in patients with advanced thyroid cancer, who had not previously received treatment with antiangiogenic agents, previously showed that axitinib was active, well tolerated and reported an investigator-assessed overall response rate (ORR) of 30\%-38\% and a median PFS of $15.0-18.1$ months $(9,10,11)$. The safety profile of axitinib was manageable, with diarrhoea, fatigue, nausea, hypertension, weight decrease and hand-foot syndrome being the most frequently reported adverse events (AEs).

We report the analysis of patients with advanced RAIrefractory DTC and locally advanced or metastatic MTC treated in Spain within a compassionate use programme (CUP) of axitinib. The study was conducted within the Spanish Task Force for Orphan and Infrequent Tumours (GETHI, Grupo Español de Tumores Huérfanos e Infrecuentes).

\section{Subjects and methods}

\section{Design and patient population}

This was a retrospective longitudinal study of patients included in the Spanish CUP of axitinib for metastatic

Table 1 Implications of the use of antiangiogenic multikinase inhibitors in the treatment of DTC and MTC.

\begin{tabular}{l} 
Current practice \\
\hline Antiangiogenic multikinase inhibitors (MKI) are \\
frequently used to treat radioactive iodine (RAI)- \\
refractory differentiated thyroid cancer (DTC) or \\
medullary thyroid cancer (MTC) that cannot be \\
managed with surgery or local therapies. Axitinib, an \\
antiangiogenic MKI currently approved for the \\
treatment of renal cell carcinoma, has not been \\
approved for the treatment of thyroid cancer
\end{tabular}

\begin{tabular}{l} 
Best practice \\
\hline Axitinib has promising clinical \\
efficacy as first-line treatment \\
of patients with refractory and \\
progressive thyroid cancer \\
regardless of tumour histology \\
(DTC or MTC) and might be \\
considered for further research \\
in these patients
\end{tabular}

The resulting gap

Better outcomes were reported when axitinib was administered as a first-line treatment, suggesting cross-resistance between MKIs in thyroid cancer and highlighting the importance of prospective sequential clinical studies 
thyroid carcinoma. Patients were included in the CUP between October 2012 and November 2014. Patients were treated with axitinib $5 \mathrm{mg}$ b.i.d. as long as it provided clinical benefit, until unacceptable toxicity occurred or disease progression was observed. Dose escalation was permitted as per the SmPC. Patients tolerating $5 \mathrm{mg}$ b.i.d. with no adverse reactions and blood pressure $\leq 150 / 90 \mathrm{mmHg}$ for two consecutive weeks could have their dose increased to $7 \mathrm{mg}$. Subsequently, patients tolerating $7 \mathrm{mg}$ b.i.d. could also have their dose increased to $10 \mathrm{mg}$ b.i.d. When dose reduction was necessary, axitinib dose was reduced to $3 \mathrm{mg}$ b.i.d. and further to $2 \mathrm{mg}$ b.i.d. Patients with papillary, follicular and medullary thyroid cancer subtypes with radiologically documented disease progression within 6 months of follow-up. Patients were allowed to be treated with axitinib as first-line therapy in this study, or following disease progression within 6 months of treatment with other MKIs were included in the study. The programme was approved by national and local regulatory authorities, and all patients signed informed consent document. Patients were evaluated following routine clinical practice every month and radiologically by CT scan, MRI or fluorodeoxyglucose PET every 8-12 weeks. RECIST v1.1 has been used to define response or progression disease regardless of the imaging technique used. Tumour marker evaluations of thyroglobulin, calcitonin and carcinoembryonic antigen (CEA) were performed every 8-12 weeks.

\section{Objectives}

We sought to define the efficacy, toxicity profile and clinical outcome of patients with advanced RAIrefractory DTC, or unresectable or metastatic MTC with radiologically documented disease progression, who received axitinib as part of the CUP in 16 referral cancer centres in Spain. The safety profile of axitinib in patients with thyroid cancer was defined according to the Common Terminology Criteria for Adverse Events (CTCAE) v4.0. The primary endpoint was determination of the objective response rate (ORR) that included partial and complete responses obtained according to Response Evaluation Criteria In Solid Tumors (RECIST) v1.1 (12). The efficacy analyses were performed on the intentto-treat (ITT) population, which included all patients included in the CUP. The secondary endpoints included safety profile, progression-free survival (PFS) defined from the time from inclusion into the CUP until an event of disease progression as determined by the investigator or death, and overall survival (OS) defined from the time from inclusion into the CUP until death. Biomarker analyses were carried out for thyroglobulin, CEA and calcitonin, and their correlation between efficacy and tumour marker levels: thyroglobulin for DTC, and calcitonin and CEA for MTC. A patient was considered to be a responder by biomarkers if there was a reduction of at least 30\% from the baseline levels of the biomarker. The biomarker analyses were carried out in the population that had biomarker information available.

\section{Statistical analysis}

Summary statistics are presented for all variables. Efficacy was assessed on the basis of investigator-assessed tumour response. Patients with unavailable response assessments were considered 'Not evaluable/missing', and for the purposes of calculating ORR they were considered nonresponders (12). Log rank of Kaplan-Meier survival estimates was used to obtain estimates of median PFS and OS, with corresponding 2-sided 95\% CIs and $\chi^{2}$ distribution used for the correlation between tumour markers and efficacy. The level of statistical significance was established at $P<0.05$. Statistical analyses were undertaken with the statistical software SPSS version 17.0 or higher.

\section{Results}

\section{Patient population}

Forty-seven patients from 16 centres in Spain received axitinib via the CUP and were included in this retrospective analysis. The mean age of the patients was $62 \pm 11$ years when starting treatment with axitinib. The median time between diagnosis and starting treatment with axitinib was 4.7 (range: 0-32.5) years. As of May 2015, 12 patients are continuing treatment with axitinib, 34 patients discontinued treatment due to disease progression and in one patient axitinib was stopped due to a Grade $4 \mathrm{AE}$.

Thirty-four (72\%) patients had DTC and 13 (28\%) had MTC histology (Table 2). The most frequent sites of metastasis were lungs, lymph nodes (both unresectable lymphadenopathies in the neck and distant lymph node metastases) and bones. Lung metastatic disease was significantly more frequent in patients with DTC (74\%) compared with patients with MTC (23\%); $P=0.003$. The baseline patient characteristics and previous treatments are shown in Table 2. 
Table 2 Patient demographics, disease characteristics and prior treatment regimens.

\begin{tabular}{|c|c|c|c|}
\hline Characteristic & Patients with DTC $(n=34)$ & Patients with MTC $(n=13)$ & All patients $(n=47)$ \\
\hline \multicolumn{4}{|l|}{ Sex, $n(\%)$} \\
\hline Male & $16(47.1)$ & $7(53.8)$ & $24(51.1)$ \\
\hline Female & $18(52.9)$ & $6(46.2)$ & $23(48.9)$ \\
\hline \multicolumn{4}{|l|}{ Age at start of treatment, years } \\
\hline Median (range) & $64(26-82)$ & $66(37-79)$ & $64(26-82)$ \\
\hline \multicolumn{4}{|l|}{ ECOG PSa (\%) } \\
\hline 0 & $10(30.3)$ & $5(38.5)$ & $15(32.6)$ \\
\hline 1 & $17(51.5)$ & $6(46.2)$ & $23(50.0)$ \\
\hline 2 & $6(18.2)$ & $2(15.4)$ & $8(17.4)$ \\
\hline \multicolumn{4}{|l|}{ Histology (\%) } \\
\hline Papillary & $15(44.1)$ & NA & \\
\hline Follicular & $10(29.4)$ & NA & \\
\hline Hürtle & $6(17.6)$ & NA & \\
\hline Poorly differentiated & $3(8.9)$ & NA & \\
\hline \multicolumn{4}{|l|}{ Location of metastases, $n(\%)$} \\
\hline Lymph nodes & $24(70.6)$ & $4(30.8)$ & $28(59.6)$ \\
\hline Lung & $25(73.5)$ & $3(23.1)$ & $28(59.6)$ \\
\hline Bone & $11(32.4)$ & $2(15.4)$ & $13(27.7)$ \\
\hline Thyroid (local unresectable disease) & $9(26.5)$ & $2(15.4)$ & $11(23.4)$ \\
\hline Liver & $1(2.9)$ & $3(23.1)$ & $4(8.5)$ \\
\hline CNS & $1(2.9)$ & 0 & $1(2.1)$ \\
\hline \multicolumn{4}{|l|}{ Previous treatments, $n(\%)$} \\
\hline Surgery & $32(94.1)$ & $10(76.9)$ & $42(89.4)$ \\
\hline Radioactive iodine & $34(100)$ & $0(0)$ & $34(72)$ \\
\hline Adjuvant EBRT & $4(11.8)$ & $2(15.4)$ & $6(12.8)$ \\
\hline Adjuvant chemotherapy & $2(5.9)$ & $1(7.7)$ & $3(6.4)$ \\
\hline$M K I^{b}$ & $21(61.8)$ & $9(69.2)$ & $30(63.8)$ \\
\hline 1 prior MKI & $14(41.2)$ & $4(30.8)$ & $18(38.3)$ \\
\hline 2 prior MKI & $6(17.6)$ & $4(30.8)$ & $10(21.3)$ \\
\hline 3 prior MKI & $1(2.9)$ & $1(7.7)$ & $2(4.3)$ \\
\hline No prior pharmacological treatment & $13(38.2)$ & $4(30.8)$ & $17(36.2)$ \\
\hline
\end{tabular}

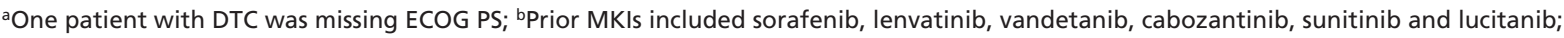
'Histology subgroup only for DTC.

DTC, differentiated thyroid cancer; EBRT, external beam radiotherapy; ECOG PS, Eastern Cooperative Group Oncology Performance Status; MKI, multikinase inhibitor; MTC, medullary thyroid cancer.

\section{Treatment}

Median treatment with axitinib was 7.1 (range: 0-24.3) months. In patients with DTC the median treatment duration was 8.1 (range: 0-22.4) months, and in patients with MTC it was 6.9 (range: 0-24.3) months. Overall, axitinib was first-line treatment for 17 (36\%) patients, second-line treatment for 18 (38\%) patients, third-line treatment for 10 (21\%) patients and fourthline treatment for 2 (4\%) patients. There were $12(25.5 \%)$ patients who had a dose reduction of axitinib from the $5 \mathrm{mg}$ b.i.d. starting dose to $3 \mathrm{mg}$ b.i.d (7 of these patients had DTC and 5 had MTC). Only in one patient the dose was increased from $5 \mathrm{mg}$ b.i.d. to $7 \mathrm{mg}$ b.i.d., with no radiological response and no additional side effects.

\section{Efficacy}

Overall, 38 of the 47 patients had data on radiologic response, but all 47 patients were included in the ITT analyses. Two (4\%) patients had a complete response as best radiologic response to treatment (Table 3). A partial response was reported in 11 (23\%) patients, stable disease in $13(28 \%)$ patients and progressive disease in 12 patients (26\%). The ORR and disease control rate (DCR=complete response + partial response + stable disease as best response by RECIST v1.1 criteria) were $28 \%$ and 55\% respectively. The results were comparable in both the DTC and MTC patient populations. When response rate was analysed according to the line of treatment, a better outcome was observed in patients who received axitinib as first-line treatment, with an ORR of 53\% compared with a $17 \%$ in patients who received axitinib as second-line treatment after progression to a previous MKI (Table 4). Similarly, DCR was reported in $82 \%$ of patients receiving firstline treatment and in 50\% of patients receiving secondline treatment.

Median PFS of the ITT population was 8.1 months (95\% CI: 4.1-12.2) (Fig. 1), with a 7.4 month (95\% CI: 
Table 3 Best response rate to axitinib in patients with advanced thyroid cancer.

\begin{tabular}{l}
\hline Besut tumour response (\%) \\
Complete response* \\
Partial response \\
Stable disease \\
Progressive disease \\
Not evaluable/missing \\
Overall response rate (\%) \\
Disease control rate (\%) \\
\hline
\end{tabular}

\begin{tabular}{c}
\hline Patients with DTC $(n=34)$ \\
\hline $2(5.9)$ \\
$8(23.5)$ \\
$8(23.5)$ \\
$8(23.5)$ \\
$8(23.5)$ \\
$10(29.4)$ \\
$18(52.9)$ \\
\hline
\end{tabular}

\begin{tabular}{c}
\hline Patients with MTC $(n=13)$ \\
\hline 0 \\
$3(23.1)$ \\
$5(38.5)$ \\
$4(30.8)$ \\
$1(7.7)$ \\
$3(23.1)$ \\
$8(61.5)$ \\
\hline
\end{tabular}

All patients $(n=47)$
$2(4.3)$
$11(23.4)$
$13(27.7)$
$12(25.5)$
$9(19.1)$
$13(27.7)$
$26(55.3)$

*The two complete responses were observed in two patients with papillary thyroid cancer with lung and lymph node metastases. Both patients were followed by CT scans and maintained complete response at the time of data analysis. In both cases, tumour burden can be considered low-medium, with lung and lymph node metastases up to $2-3 \mathrm{~cm}$ of maximum diameter.

DTC, differentiated thyroid cancer; MTC, medullary thyroid cancer.

3.1-11.8) median PFS in patients with DTC and 9.4 months (95\% CI: 4.8-13.9) in patients with MTC. Similarly, median PFS was significantly better in patients who had received fewer prior lines of treatment. Therefore the PFS was 13.6 months (95\% CI: 8.5-18.6) in patients who received axitinib as first-line, 10.6 months (95\% CI: 6.8-14.3) in second-line and 3.6 months (95\% CI: 2.0-5.2) in third-line treatment. No statistically significant differences were observed regarding histology and treatment benefit in function of treatment line $(P=0.331)$.

The median OS was 19.7 months (95\% CI: 16.6-22.8) in the overall population and was comparable in the DTC (20.7 months (95\% CI: 16.5-24.9)) and MTC (18.9 months (95\% CI: 18.4-19.4)) populations. A significantly improved median OS was also observed in patients with fewer lines of previous treatment; indeed median OS had only been reached in patients receiving third-line treatment (6.4 months).

\section{Biomarker analyses}

Thyroglobulin levels were available in 22 of the 34 patients with DTC, with a response in 15 patients (68\%). Thyroglobulin was included in the analysis when anti-thyroglobulin antibodies were not present. No analysis of variations in anti-thyroglobulin antibodies was performed due to the low number of cases. CEA and calcitonin expression were available in 10 patients with MTC; there was a CEA biomarker response in 5 patients (50\%) and a calcitonin biomarker response in 7 patients (70\%). The Kappa index correlation observed between $>30 \%$ biomarker reduction and ORR/DCR was 0.05/0.68 for thyroglobulin in patients with DTC, 0.14/0.05 for CEA and calcitonin in patients with MTC. Therefore no statistically significant correlation was observed between radiological and biological response.

\section{Safety}

The most frequently reported AEs were asthenia (53\% $(n=25))$, diarrhoea $(36 \%(n=17))$, hypertension $(32 \%$ $(n=15))$ and mucositis $(30 \%(n=14))$ (Table 5). The frequency and type of AE were comparable in the DTC and MTC populations. The majority of the AEs reported were of Grade 1 or 2, with few Grade 3 AEs reported. There was only 1 Grade 4 AE reported, a cardiac toxicity in a patient with DTC. The cardiac toxicity was an acute myocardial

Table 4 Best response rate to axitinib in patients with advanced thyroid cancer according to the line of treatment.

\begin{tabular}{l}
\hline Best tumour response (\%) \\
Complete response \\
Partial response \\
Stable disease \\
Progressive disease \\
Not evaluable/missing \\
Overall response rate (\%) \\
Disease control rate $(\%)$ \\
\hline
\end{tabular}

\begin{tabular}{c}
\hline 1st line axitinib $(n=17)$ \\
\hline $1(5.9)$ \\
$8(47.1)$ \\
$5(29.4)$ \\
0 \\
$3(17.6)$ \\
$9(52.9)$ \\
$14(82.4)$ \\
\hline
\end{tabular}

\begin{tabular}{c}
\hline 2nd line axitinib $(n=18)$ \\
\hline $1(5.6)$ \\
$2(11.1)$ \\
$6(33.3)$ \\
$4(22.2)$ \\
$5(27.8)$ \\
$3(16.7)$ \\
$9(50.0)$ \\
\hline
\end{tabular}

\begin{tabular}{c}
\hline 3rd line axitinib $(n=10)$ \\
\hline 0 \\
0 \\
$2(20.0)$ \\
$7(70.0)$ \\
$1(10.0)$ \\
0 \\
$2(20.0)$ \\
\hline
\end{tabular}

There were 2 patients who received axitinib as $4^{\text {th }}$ line multikinase inhibitor. One patient reported a partial response and one patient had progressive disease.

DTC, differentiated thyroid cancer; MTC, medullary thyroid cancer. 
A

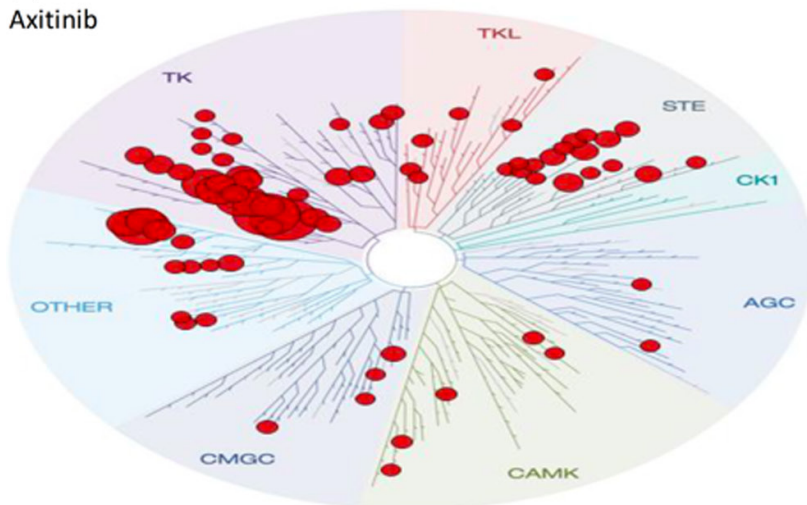

B

Lenvatinib

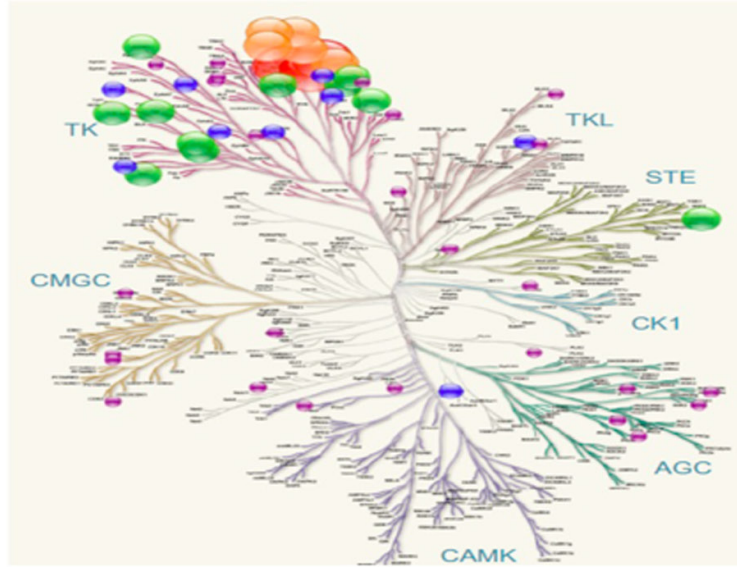

C

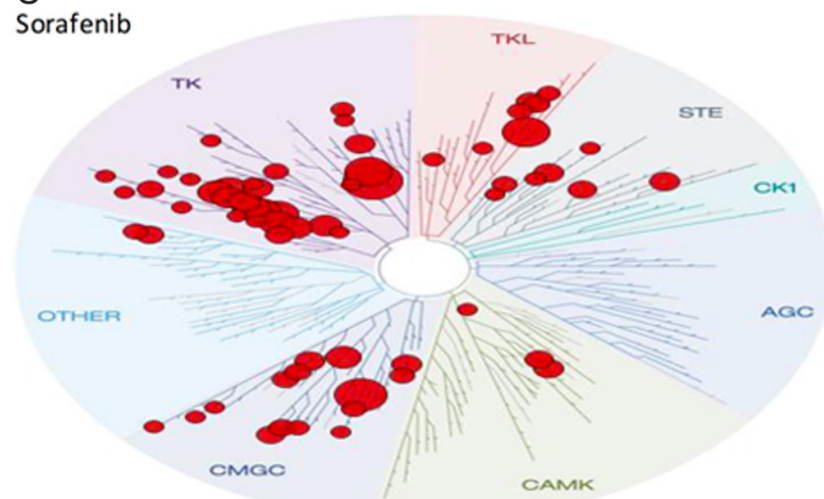

D

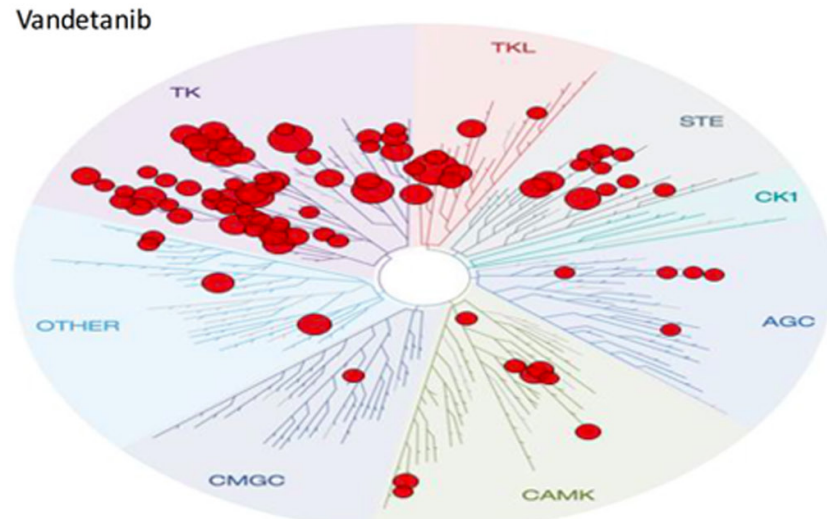

Figure 1

Kinome analysis of axitinib (A), lenvatinib (B), sorafenib (C) and vandetanib (D). Illustration reproduced courtesy of Cell Signaling Technology, Inc. (www.cellsignal.com). Adapted from (25) and image generated using TREEspot Software Tool and reprinted with permission from KINOMEscan, a division of DiscoveRx Corporation, DISCOVERX CORPORATION 2010.

infarction and ventricular systolic dysfunction, recovered after specific therapy and drug discontinuation.

\section{Discussion}

We have evaluated the clinical use of axitinib either in treatment-naive or MKI-pretreated patients with advanced thyroid cancer of DTC or MTC histology in the setting of a CUP and showed that first-line axitinib has activity with a promising ORR, DCR and PFS, as well as a manageable safety profile. Comparing clinical trial results in thyroid cancer with observations made in a CUP is difficult but may provide information on the efficacy and toxicity of axitinib outside the trial setting.

The overall efficacy results obtained in the setting of a CUP were comparable to what has been reported in clinical trials with axitinib. Two phase II studies have evaluated axitinib in patients with advanced DTC or MTC. In one phase II study in 60 patients with all histological subtypes of advanced thyroid cancer in which iodine- 131 failed to control the disease or was not the appropriate therapy, axitinib $5 \mathrm{mg}$ b.i.d. showed an investigator-assessed ORR of 30\% (95\% CI: 18.9-43.2\%) and median PFS of 18.1 months (95\% CI: 12.1 to not estimable) (7). Long-term outcomes reported an ORR of $38 \%$ and DCR of $68 \%$. After an estimated median follow-up of 34 months, median PFS and OS were 15 months and 35 months respectively (8). In a second phase II study, axitinib administration of $5 \mathrm{mg}$ b.i.d. for a median duration of 12.9 months in 52 patients with metastatic or unresectable, locally advanced RAI-refractory DTC or MTC resulted in an ORR of 35\% (95\% CI: 22-49\%), a median PFS of 16.1 months (95\% CI: 14.6-21.8 months) and median OS of 27.2 months (95\% CI: 14.6-40.1 months), with a greater axitinib 
Table 5 Safety profile of axitinib in patients with advanced thyroid cancer.

\begin{tabular}{lcc}
\hline & \multicolumn{2}{c}{ Patients with DTC $(n=34)$} \\
\cline { 3 - 3 } \cline { 3 - 3 } \cline { 3 - 3 } Asthenia & All grades & Grade $\geq 3$ \\
Diarrhoea & $15(44.1)$ & 0 \\
Hypertension & $12(35.3)$ & $2(5.9)$ \\
Mucositis & $11(32.4)$ & $1(2.9)$ \\
Hand-foot syndrome & $12(35.3)$ & 0 \\
Rash & $5(14.7)$ & 0 \\
Anorexia & $3(8.8)$ & 0 \\
Abdominal pain & $5(14.7)$ & $3(8.8)$ \\
Weight loss & $5(14.7)$ & 0 \\
Nausea & $2(5.9)$ & $1(2.9)$ \\
Vomiting & $1(2.9)$ & 0 \\
Anaemia & $1(2.9)$ & 0 \\
Cardiac toxicity & $2(5.9)$ & $1(2.9)$ \\
Headache & $1(2.9)$ & $1(2.9)$ \\
Peripheral oedema & 0 & 0 \\
Hypercholesterolaemia & 0 & 0 \\
Hyperglycaemia & $1(2.9)$ & 0 \\
Cough & $1(2.9)$ & 0 \\
Other toxicities & 0 & 0 \\
\hline
\end{tabular}

\begin{tabular}{lll}
\hline \multicolumn{2}{c}{ Patients with MTC $(n=13)$} \\
\cline { 1 - 1 } All grades & & Grade $\geq 3$ \\
\cline { 1 - 1 } $10(76.9)$ & & 0 \\
$5(38.5)$ & & 0 \\
$4(30.8)$ & & 0 \\
$2(15.4)$ & & $1(7.7)$ \\
$4(30.8)$ & & $1(7.7)$ \\
$4(30.8)$ & & 0 \\
$1(7.7)$ & & 0 \\
$1(7.7)$ & & 0 \\
$2(15.4)$ & & 0 \\
$2(15.4)$ & & 0 \\
$2(15.4)$ & & 0 \\
0 & 0 \\
$1(7.7)$ & $1(7.7)$ \\
$1(7.7)$ & 1 \\
$1(7.7)$ & 0 \\
0 & 0 \\
0 & 0 \\
$1(7.7)$ & 0 \\
$1(7.7)$ & 0
\end{tabular}

\begin{tabular}{cll}
\hline \multicolumn{2}{c}{ All patients $(n=47)$} \\
\cline { 1 - 1 } All grades & Grade $\geq 3$ \\
\cline { 1 - 1 } $25(53.2)$ & & 0 \\
$17(36.2)$ & & $2(4.3)$ \\
$15(31.9)$ & & $1(2.1)$ \\
$14(29.8)$ & & $1(2.1)$ \\
$9(19.1)$ & & $1(2.1)$ \\
$7(14.9)$ & & 0 \\
$6(12.8)$ & & $3(6.4)$ \\
$6(12.8)$ & & 0 \\
$4(8.5)$ & & $1(2.1)$ \\
$3(6.4)$ & 0 \\
$3(6.4)$ & 0 \\
$2(4.3)$ & $1(2.1)$ \\
$2(4.3)$ & $2(4.3)$ \\
$1(2.1)$ & $1(2.1)$ \\
$1(2.1)$ & 0 \\
$1(2.1)$ & 0 \\
$1(2.1)$ & 0 \\
$1(2.1)$ & 0 \\
$4(8.5)$ & $1(2.1)$
\end{tabular}

DTC, differentiated thyroid cancer; MTC, medullary thyroid cancer.

exposure correlating with a longer median PFS (9). Importantly, quality of life was maintained during treatment with axitinib and no significant deterioration in symptoms or interference in daily life caused by symptoms was reported.

Interestingly, when considering only the results from patients who received axitinib as first-line treatment, the ORR (53\%) and DCR (82\%) were higher in the CUP compared with the phase II clinical studies (ORR 38\% and DCR 68\%), albeit a lower median PFS (13.6 months in the CUP vs 18.1 months). Efficacy outcomes of axitinib in the CUP significantly decreased when patients had received prior MKIs. To our knowledge this is the first time that resistance to sequential antiangiogenic MKI therapy in advanced thyroid cancer is suggested. This hypothesis has not been reported with other MKIs in clinical trials (13, $14,15,16,17)$ or in clinical practice $(18,19)$. Furthermore, the pivotal phase III study of axitinib in RCC also showed in the subgroup analyses that PFS was significantly shorter in those patients treated with axitinib after progression to sunitinib compared with those who received axitinib after progression to cytokines (20). The different spectrum of MKI activity could explain how these drugs are active in different treatment lines (Fig. 2) (3). Axitinib is one of the best VEGFR inhibitors in clinical development regarding $\mathrm{IC}_{50}$ values of inhibition of VEGFR1-3, even at a below nanomolar level. However axitinib has no additional inhibition of interesting targets in thyroid cancer, such as the translocation of the rearranged during transfection tyrosine kinase receptor in papillary thyroid cancer (RET/PTC), BRAF, c-MET, epidermal growth factor receptor (EGFR) or fibroblast growth factor receptors (FGFR1-4). The approved MKIs for refractory thyroid cancer have

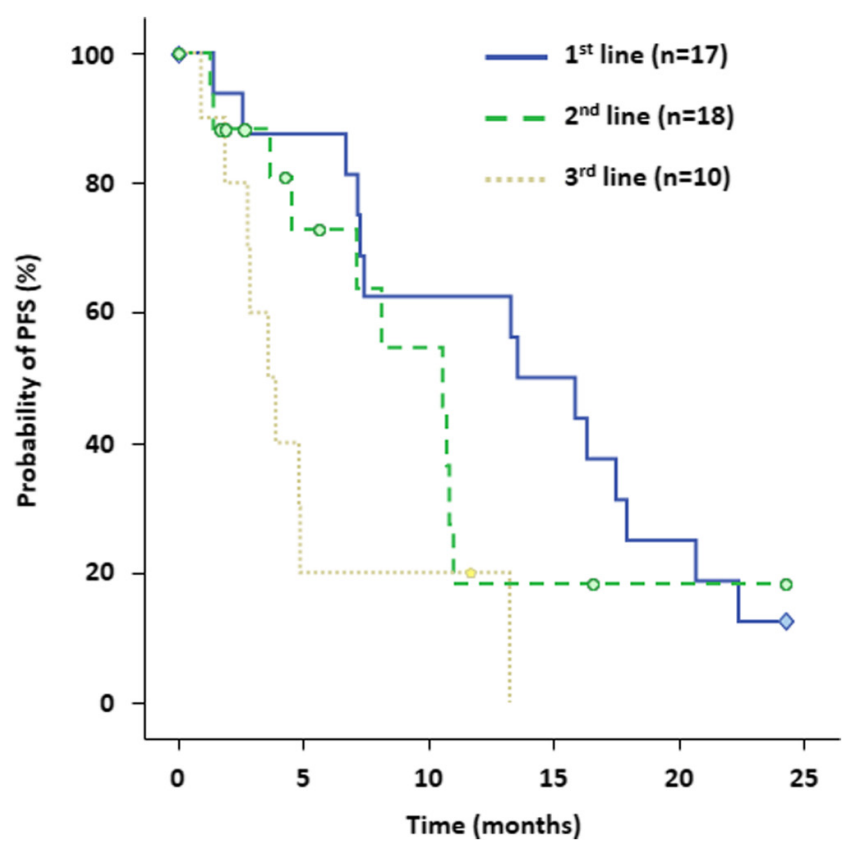

Figure 2

Kaplan-Meier curves indicating progression-free survival (PFS) of axitinib in patients with advanced thyroid cancer according to line of treatment. 
this additional activity to other targets that could revert the resistance to antiangiogenesis inhibition and could work after progression to a prior MKI, clearly shown for cabozantinib and lenvatinib in their phase III studies. The lack of these additional effects could be behind the resistance observed with axitinib after progression to other MKIs in thyroid cancer.

In our analysis, axitinib was generally well tolerated with a safety profile according to what was expected from previous clinical trials. Most common AEs of axitinib were mild to moderate in severity. They included diarrhoea, hypertension, fatigue, dysphonia, nausea, decreased appetite and hand-foot syndrome (6). Most AEs are manageable with symptomatic treatment and dose reductions. Approximately one-quarter of the patients underwent a dose reduction to manage toxicity, and one patient required treatment discontinuation due to a Grade 4 cardiac toxicity. In a phase II study of axitinib in thyroid cancer, $40 \%$ of the patients had a dose reduction and four treatment-related AEs led to permanent axitinib discontinuation. The overall safety profile reported in this study is consistent with what had been previously reported in patients with metastatic RCC, and clinical study data confirm a toxicity profile comparable to that of other agents in the class (21).

We acknowledge that our study has important weaknesses, considering the retrospective design and the characteristic limitations derived from a CUP such as the heterogeneity in patients and disease characteristics, the local investigator-assessed efficacy, and the worse performance status of patients who qualify for a CUP compared with those included in clinical trials. Furthermore, it is impossible to draw definitive conclusions on the activity of axitinib in patients with metastatic thyroid cancer; however our results provide further support for evaluation of axitinib in prospective, randomized clinical trials. There was an encouraging clinical benefit when administrating axitinib in firstline therapy that progressively decreased in successive treatment lines, conditioning the design of future sequential clinical trials.

As noted earlier, cabozantinib and vandetanib are approved for unresectable MTC, and sorafenib and lenvatinib for RAI-refractory DTC. The ORR and median PFS in the phase III studies leading to regulatory approval was $28 \%$ and 11.2 months in the cabozantinib arm in the EXAM trial (15) and $45 \%$ and 30.5 months in the vandetanib arm in the ZETA trial (22) in patients with MTC. In patients with DTC, the ORR was $12.2 \%$ and the median PFS was 10.8 months in the sorafenib arm in the DECISION trial (23) and $64.8 \%$ and 18.3 months in the lenvatinib arm in the SELECT trial (17), with $65.6 \%$ and 18.7 months in patients receiving first-line lenvatinib.

In conclusion, axitinib showed promising efficacy and safety profile in first-line treatment in both patients with DTC and MTC histologies. However the efficacy of axitinib significantly decreases in second and successive lines after progression to prior systemic therapy with MKIs raising the hypothesis of anti-angiogenic crossresistance in thyroid cancer setting. Our clinical observation warrants careful consideration of the next steps in the treatment of patients with advanced thyroid cancer. Currently, many clinical trials are assessing the efficacy of different MKIs after progression to approved drugs in both histologies (sorafenib and lenvatinib for DTC and vandetanib and cabozantinib for MTC), both as monotherapy and in combination with other targeted agents (24). The correct design of these clinical trials will help define the successful sequential treatment schedules for these patients and minimize the exposure to inactive drugs in more advanced stages where life expectancy is significantly reduced.

Declaration of interest

J C and J A have received honoraria as speaker or advisory role by Bayer, Pfizer and Eisai. E G has received honoraria as speaker or advisory role by Bayer, Astra Zeneca, Pfizer and Eisai, and has received research grant from Pfizer. T R C has received lecturing fees by Pfizer and Astra Zeneca, and consulting fees by Bayer and Eisai. J T, J L M, S G A, C Z, O R, U B, M D P, B G $A, A L A, J M M, I P, J J R, N P, E C, I M$ and J J $G$ have no conflicts of interests.

Funding

The CUP of axitinib was funded by Pfizer, Spain.

\section{Acknowledgements}

The authors wish to thank all the patients who participated in the study and their families and caregivers. The authors also thank Aurora O'Brate who provided medical writing services, on behalf of Pfizer, Spain. Special acknowledgement is given to GETHI for support during data collection (online database), for help promoting the CUP, and for providing logistical support.

\section{References}

1 Ferlay J, Soerjomataram I, Ervik M, Dikshit R, Eser S, Mathers C, Rebelo M, Parkin DM, Forman D, Bray F et al. GLOBOCAN 2012 v1.0, cancer incidence and mortality worldwide: IARC CancerBase No. 11 (Internet). Lyon, France: International Agency for Research on Cancer, 2013. (Available at: http://globocan.iarc.fr). Accessed on 20 April 2015.

2 Klein M, Picard E, Vignaud JM, Marie B, Bresler L, Toussaint B, Weryha G, Duprez A, Leclère J et al. Vascular endothelial growth factor gene and protein: strong expression in thyroiditis 
and thyroid carcinoma. Journal of Endocrinology $199916 \mathbf{1}$ 41-49. (doi:10.1677/joe.0.1610041)

3 Gruber JJ \& Colevas AD. Differentiated thyroid cancer: focus on emerging treatments for radioactive iodine-refractory patients. Oncologist 201520 113-126. (doi:10.1634/theoncologist.2014-0313)

4 Trigo JM, Capdevila J, Grande E, Grau J, Lianes P for the Spanish Society for Medical Oncology. Thyroid cancer SEOM clinical guidelines. Clinical and Translational Oncology 201416 1035-1042. (doi:10.1007/s12094-014-1224-4)

5 Tuttle RM, Haddad RI, Ball DW, Byrd D, Dickson P, Duh QY, Ehya H, Haymart M, Hoh C, Hunt JP et al. Thyroid carcinoma, version 2. 2014. Journal of the National Comprehensive Cancer 201412 1671-1680. (doi:10.6004/jnccn.2014.0169)

6 European Medicines Agency. Inlyta ${ }^{\circledR}$ (axitinib) summary of product characteristics. Pfizer Inc, 2015. (Available at http://www.ema.europa. eu/docs/en_GB/document_library/EPAR_-_Product_Information/ human/002406/WC500132188.pdf). Accessed on 20 April 2015.

7 McNamara MG, Le LW, Horgan AM, Aspinall A, Burak KW, Dhani N, Chen E, Sinaei M, Lo G, Kim TK et al. A phase II trial of second-line axitinib following prior antiangiogenic therapy in advanced hepatocellular carcinoma. Cancer 2015121 1620-1627 (doi:10.1002/cncr.29227)

8 Schiller JH, Larson T, Ou SH, Limentani S, Sandler A, Vokes E, Kim S, Liau K, Bycott P, Olszanski AJ et al. Efficacy and safety of axitinib in patients with advanced non-small-cell lung cancer: results from a phase II study. Journal of Clinical Oncology 200927 3836-3841 (doi:10.1200/JCO.2008.20.8355)

9 Cohen EE, Rosen LS, Vokes EE, Kies MS, Forastiere AA, Worden FP, Kane MA, Sherman E, Kim S, Bycott P et al. Axitinib is an active treatment for all histologic subtypes of advanced thyroid cancer: results from a phase II study. Journal of Clinical Oncology 200826 4708-4713. (doi:10.1200/JCO.2007.15.9566)

10 Cohen EE, Tortorici M, Kim S, Ingrosso A, Pithavala YK \& Bycott P. A phase II trial of axitinib in patients with various histologic subtypes of advanced thyroid cancer: long-term outcomes and pharmacokinetic/pharmacodynamic analyses. Cancer Chemotherapy and Pharmacology 201474 1261-1270. (doi:10.1007/s00280-014-2604-8)

11 Locati LD, Licitra L, Agate L, Ou SH, Boucher A, Jarzab B, Qin S, Kane MA, Wirth LJ, Chen C et al. Treatment of advanced thyroid cancer with axitinib: phase 2 study with pharmacokinetic/ pharmacodynamic and quality-of-life assessments. Cancer 2014120 2694-2703. (doi:10.1002/cncr.28766)

12 Eisenhauer EA, Therasse P, Bogaerts J, Schwartz LH, Sargent D, Ford R, Dancey J, Arbuck S, Gwyther S, Mooney M et al. New response evaluation criteria in solid tumours: revised RECIST guideline (version 1.1). European Journal of Cancer 200945 228-247. (doi:10.1016/j.ejca.2008.10.026)

13 Bible KC, Suman VJ, Molina JR, Smallridge RC, Maples WJ, Menefee ME, Rubin J, Sideras K, Morris JC 3rd, McIver B, Burton JK Endocrine Malignancies Disease Oriented Group, Mayo Clinic Cancer Center, Mayo Phase 2 Consortium et al. Efficacy of pazopanib in progressive, radioiodine-refractory, metastatic differentiated thyroid cancers: results of a phase 2 consortium study. Lancet Oncology 201011 962-972. (doi:10.1210/jc.2013-3713)

14 Cabanillas ME, Schlumberger M, Jarzab B, Martins RG, Pacini F, Robinson B, McCaffrey JC, Shah MH, Bodenner DL, Topliss D et al. A phase 2 trial of lenvatinib (E7080) in advanced, progressive, radioiodine-refractory, differentiated thyroid cancer: a clinical outcomes and biomarker assessment. Cancer 20151212749 2756. (doi:10.1002/cncr.29395)

15 Elisei R, Schlumberger MJ, Müller SP, Schöffski P, Brose MS, Shah MH, Licitra L, Jarzab B, Medvedev V, Kreissl MC et al. Cabozantinib in progressive medullary thyroid cancer. Journal of Clinical Oncology 2013 31 3639-3646. (doi:10.1200/JCO.2012.48.4659)

16 Newbold K, Elisei R, Taylor MH, Krzyzanowska MK, Shah MH, Hoff AO, Robinson B, Dutcus C, Song J \& Habra MA. Efficacy and safety of lenvatinib for the treatment of patients with ${ }^{131} \mathrm{I}$-refractory differentiated thyroid cancer with and without prior VEGF-targeted therapy. Journal of Clinical Oncology 201533 (Supplement) abstract 6013. (doi:10.1200/jco.2015.33.15_suppl.6013)

17 Schlumberger M, Tahara M, Wirth LJ, Robinson B, Brose MS, Elisei R, Habra MA, Newbold K, Shah MH, Hoff AO et al. Lenvatinib versus placebo in radioiodine-refractory thyroid cancer. New England Journal of Medicine 2015372 621-630. (doi:10.1056/NEJMoa1406470)

18 Capdevila J, Iglesias L, Halperin I, Segura A, Martínez-Trufero J, Vaz MÁ, Corral J, Obiols G, Grande E, Grau JJ et al. Sorafenib in metastatic thyroid cancer. Endocrine-Related Cancer 201219 209-216. (doi:10.1530/ERC-11-0351)

19 Capdevila J, Martinez Trufero J, Dalmau E, Alvarez-Escola C, Beltran M, Duran M, Gallegos I, Manzano JL, Mesia R, Pajares I et al. Retrospective analysis of the safety and efficacy of vandetanib as systemic treatment for patients with advanced and progressive medullary thyroid cancer (MTC). Journal of Clinical Oncology 201432 (Supplement) abstract e17015. (doi:10.1200/jco.2014.32.15_suppl.e17015)

20 Rini BI, Escudier B, Tomczak P, Kaprin A, Szczylik C, Hutson TE, Michaelson MD, Gorbunova VA, Gore ME, Rusakov IG et al. Comparative effectiveness of axitinib versus sorafenib in advanced renal cell carcinoma (AXIS): a randomised phase 3 trial. Lancet 2011 378 1931-1939. (doi:10.1016/S0140-6736(11)61613-9)

21 Gunnarsson O, Pfanzelter NR, Cohen RB \& Keefe SM. Evaluating the safety and efficacy of axitinib in the treatment of advanced renal cell carcinoma. Cancer Management and Research 20157 65-73. (doi:10.2147/CMAR.S74202)

22 Wells SA Jr, Robinson BG, Gagel RF, Dralle H, Fagin JA, Santoro M, Baudin E, Elisei R, Jarzab B, Vasselli JR et al. Vandetanib in patients with locally advanced or metastatic medullary thyroid cancer: a randomized, double-blind phase III trial. Journal of Clinical Oncology 201230 134-141. (doi:10.1200/JCO.2011.35.5040)

23 Brose MS, Nutting CM, Jarzab B, Elisei R, Siena S, Bastholt L, de la Fouchardiere C, Pacini F, Paschke R, Shong YK et al. DECISION investigators Sorafenib in radioactive iodine-refractory, locally advanced or metastatic differentiated thyroid cancer: a randomised, double-blind, phase 3 trial. Lancet 2014384 319-328. (doi:10.1016/ S0140-6736(14)60421-9)

24 Brose MS, Troxel AB, Yarchoan M, Cohen AB, Harlacker K, Dyanick NA, Posey ZA, Makani R, Prajapati P, Zifchak LM et al. A phase II study of everolimus (E) and sorafenib (S) in patients (PTS) with metastatic differentiated thyroid cancer who have progressed on sorafenib alone. Journal of Clinical Oncology 201533 (Supplement) abstract 6072. (doi:10.1200/jco.2015.33.15_suppl.6072)

25 Stjepanovic N \& Capdevila J. Multikinase inhibitors in the treatment of thyroid cancer: specific role of lenvatinib. Biologics 2014 8 129-139.
Received 23 March 2017

Revised version received 25 May 2017

Accepted 29 June 2017 